\title{
SEROPREVALENCIA DE CISTICERCOSIS PORCINA EN CASERÍOS RURALES DEL DEPARTAMENTO DE TUMBES, PERÚ
}

\author{
Seroprevalence of Porcine Cysticercosis in Rural Villages of the \\ Department of Tumbes, Peru
}

\section{Bruno García A. ${ }^{1}$, Armando González Z. ${ }^{1,2}$, Teresa López U. ${ }^{3}$, Arnaldo Alvarado S. ${ }^{4}$}

\section{RESUMEN}

Se determinó la seroprevalencia de cisticercosis porcina en caseríos de la zona de Tumbes, Perú. Se evaluó el 97\% (1872/1927) del total de cerdos mayores a 7 meses de edad, provenientes de 17 caseríos rurales de la provincia de Tumbes, departamento de Tumbes, mediante la prueba de Electroinmunotransferencia. Además, se evaluó las variables caserío de procedencia, edad y sexo para determinar si existía asociación respecto a la presentación de la enfermedad, mediante la prueba de Chi Cuadrado y el análisis de Regresión Logística. La seroprevalencia corregida fue de $45 \pm 2.3 \%$. Los caseríos que presentaron las seroprevalencias más altas fueron La Capitana, Carretas y Rica Playa (70 $\pm 7.8,69 \pm 12.3$ y $64 \pm 6.8 \%$, respectivamente). Los seroprevalencia en cerdos mayores de 12 meses fue de $49 \pm 3.1 \%$ en tanto que en cerdos de 8 a 12 meses fue de $40 \pm 3.3 \%$ $(\mathrm{p}<0.05)$. No se encontró diferencias estadísticas por efecto del sexo. Los resultados demuestran que la cisticercosis porcina constituye un serio problema de salud pública para los caseríos estudiados.

Palabras clave: Taenia solium, cisticercosis porcina, seroprevalencia, Tumbes

\section{AbSTRACT}

The porcine cysticercosis seroprevalence was determined in rural villages of Tumbes, Peru. Samples from 97\% (1872/1927) of pigs, older than 7 months of age, from 17 rural villages of Tumbes province, Peru, were analyzed by the Electroimmunotransfer test. Also, village of origin, age, and sex were assessed to determine whether there was association with regard to the presentation of the disease by the Chi square test and logistic regression analysis. Seroprevalence was $45 \pm 2.3 \%$. Villages with higher seroprevalences were La Capitana, Carretas, and Rica Playa $(70 \pm 7.8,69 \pm 12.3$, and $64 \pm$

\footnotetext{
${ }^{1}$ Laboratorio de Medicina Veterinaria Preventiva, ${ }^{3}$ Laboratorio de Microbiología y Parasitología Veterinaria, ${ }^{4}$ Laboratorio de Patología Clínica y Biología Molecular, Facultad de Medicina Veterinaria, Universidad Nacional Mayor de San Marcos, Lima

${ }^{2}$ E-mail: agonzale@jhsph.edu
} 
$6.8 \%$ respectively). Seroprevalence in pigs older than 12 months of age was $49 \pm 3.1 \%$, while in pigs 8 to 12 months of age was $40 \pm 3.3 \%$ ( $p<0.05$ ). There was no statistical difference due to sex. The results show that porcine cysticercosis is a serious public health problem for the villages surveyed.

Key words: Taenia solium, porcine cysticercosis, seroprevalence, Tumbes

\section{INTRODUCCIÓN}

La cisticercosis porcina es una zoonosis parasitaria producida por la forma larvaria de la Taenia solium, denominada Cysticercus cellulosae. El hospedador definitivo es el hombre, quien alberga la forma adulta en el intestino, y el hospedador intermediario natural es el cerdo, que se infecta ingiriendo huevos o proglótidos (Náquira, 1999; García y González, 2000). Esta enfermedad es importante en salud pública, debido a que el hombre, además de padecer la teniasis, también puede desarrollar la cisticercosis, cuando de manera accidental consume huevos de tenia. La neurocisticercosis, la forma más común de la cisticercosis en el hombre, es ocasionada por el alojamiento de la larva en el sistema nervioso central, donde puede ocasionar serias discapacidades neurológicas (Del Brutto, 1999).

La distribución del Cysticercus cellulosae es mundial y coincide con la distribución de la infección con la tenia adulta (Acha y Szyfres, 2003). La cisticercosis porcina ha perdido importancia en países donde se practica la crianza intensiva y existen servicios de inspección veterinaria adecuados, pero sigue constituyendo un serio problema sanitario en áreas rurales con niveles sociales e higiénicos deficientes de África subsahariana, Asia y Latinoamérica, a excepción de las poblaciones que por razones religiosas no consumen carne de cerdo (Cordero del Campillo e Hidalgo Argüello, 1999).

La presencia de la enfermedad está asociada a varios factores. La crianza libre de cerdos, la falta de hábitos de higiene personal, la carencia de letrinas u otro sistema de disposición de excretas, así como el mal uso de estas, permiten que los cerdos tengan acceso a las heces humanas. Además, el problema se agrava cuando la población no cuenta con un fácil acceso a la atención médica especializada (OPS, 1992). Esta enfermedad ocasiona grandes pérdidas en la economía de los pequeños criadores de cerdos, causadas por la merma del valor comercial de los animales infectados y por el decomiso en los camales de las carcasas parasitadas; situación que ocasiona que exista un comercio informal de cerdos infectados por cisticercos (CWGP, 1993).

En las áreas rurales, las prevalencias de cisticercosis en cerdos son mayores que las de la teniasis. Estas características epidemiológicas de la enfermedad en el cerdo hacen que la prevalencia de la cisticercosis porcina sea un importante indicador de la infección con $T$. solium en humanos. Además, permiten llevar a cabo estrategias de control que son aceptadas por los pobladores de las zonas rurales. Entre las principales se tiene a la vacunación (Sciutto et al., 1998), el uso de antiparasitarios con diferentes niveles de efectividad (González et al., 1999; González, 2002), y la educación de la población (Sartí et al., 1997).

El objetivo de este trabajo fue determinar la seroprevalencia de la cisticercosis porcina en caseríos rurales del departamento de Tumbes, mediante el uso de la prueba de Electroinmuno Transferencia Blot (EITB), a fin de poder llevar a cabo las medidas necesarias para el control de la enfermedad. 


\section{MateiRIALeS y Métodos}

\section{Lugar de Estudio}

El estudio fue realizado en los caseríos rurales de San Francisco, Malval, El Rodeo, y Cristales ubicados en el distrito de Corrales, y en los caseríos de Pechichal, San Jacinto, Plateros, Santa Rosa, La Peña, Francos, Vaquería, El Oidor, Casa Blanqueada, Carretas, Higuerón, La Capitana y Rica Playa, ubicados en el distrito de San Jacinto. Ambos distritos pertenecen a la provincia de Tumbes, departamento de Tumbes. Todos los caseríos se encuentran ubicados en el margen occidental del río Tumbes y el acceso a ellos es a través de una carretera afirmada.

La población del distrito de Corrales es de 20984 habitantes, donde el área rural solo representa el $5.9 \%$ de la población total. El distrito de San Jacinto tiene una población de 7979 habitantes, donde el área rural representa el 51.4\% del total (INEI, 2007). La provincia de Tumbes presenta una altitud promedio de $7 \mathrm{msnm}$, clima semitropical y con sol permanente durante todo el año. La temperatura media anual es de $24{ }^{\circ} \mathrm{C}$ (Portal Municipal Tumbes, 2008).

\section{Animales y Muestras}

Se trabajó con la totalidad de la población porcina de cada uno de los caseríos rurales, exceptuando las hembras preñadas y animales menores a siete meses. Los cerdos fueron identificados con aretes y se registró el sexo, edad y vivienda a la que pertenecen. Se tomó muestras de sangre de la vena cava anterior empleando tubos al vacío de $8.5 \mathrm{ml}$ con gel separador (BD Vacutainer®), se refrigeraron y el suero se obtuvo por centrifugación dentro de las dos horas. Los sueros resultantes se llevaron a la ciudad de Lima manteniendo la cadena de frío con hielo seco, donde se almacenaron en congelación a $-20^{\circ} \mathrm{C}$.
En el análisis de datos se tomó en cuenta a la población porcina mayor a siete meses de edad, para asegurar que se encuentren libres de anticuerpos maternales contra cisticercosis porcina, dado su interferencia en la interpretación de los resultados al sobrestimar los datos de seroprevalencia (Ccama $e t$ al., 2003).

\section{Análisis de Muestras}

Las muestras recolectadas fueron procesadas mediante la técnica de EITB (Tsang et al., 1989; Gavidia, 1993), para establecer la presencia de anticuerpos contra la forma larvaria de Taenia solium. La lectura final de la prueba da siete bandas diagnósticas. Para el estudio se consideraron positivos aquellos animales que presentaron reacción a cualquiera de estas bandas: glicoproteína (GP) 50 kilodalton (kDa), GP 42-39 kDa, GP $24 \mathrm{kDa}, \mathrm{GP} 21 \mathrm{kDa}, \mathrm{GP} 18 \mathrm{kDa}$, GP $14 \mathrm{kDa}$, GP 13 kDa, (Tsang et al., 1989).

\section{Análisis de Datos}

Se determinó la prevalencia de la enfermedad mediante la siguiente fórmula, donde $p=$ Prevalencia a la prueba, y $n=$ tamaño muestral.

$$
p ? \frac{N^{o} \text { positivos }}{n} ? 100
$$

La prevalencia corregida se determinó mediante la siguiente fórmula (Ahlbom y Norell, 1990), donde $P c=$ Prevalencia corregida, $p=$ prevalencia a la prueba, $?=$ sensibilidad 98\% (González et al., 1990), y ?= especificidad 100\% (González et al., 1990).

$$
P_{c} ? \frac{p ? ? ? 1}{? ? ? ? 1}
$$

Los resultados se expresaron con un intervalo de confianza de $95 \%$, determinado a través de la siguiente fórmula (Armitage y Berry, 1987), donde $p=$ prevalencia encontrada (expresada en proporción), $z=1.96, \mathrm{y}$ $n=$ tamaño muestral.

$$
\text { I.C. ? } p ? z \sqrt{p ? ! 1 ? p ? / n}
$$


Cuadro 1. Seroprevalencia de cisticercosis porcina en animales mayores de siete meses de edad en la provincia de Tumbes, Perú, según número de bandas reactivas a la prueba de EITB (2006)

\begin{tabular}{|c|c|c|c|}
\hline $\begin{array}{c}\mathrm{N}^{\circ} \\
\text { bandas }\end{array}$ & $\begin{array}{c}\text { Positivo } \\
(\mathrm{n}=1872)\end{array}$ & $\begin{array}{c}\text { Prevalencia } \\
(\%)\end{array}$ & $\begin{array}{l}\text { I.C. }{ }^{1} \\
(\%)\end{array}$ \\
\hline 1 & 234 & 13 & 1.5 \\
\hline 2 & 158 & 9 & 1.3 \\
\hline 3 & 380 & 21 & 1.8 \\
\hline$>3$ & 51 & 3 & 0.7 \\
\hline Total & 823 & 45 & 2.3 \\
\hline
\end{tabular}

Se evaluó el efecto de las variables sexo, edad y caserío sobre la presencia de la enfermedad, mediante la prueba de Chi Cuadrado y el Análisis de Regresión Logística, utilizando el programa estadístico STATA 9.2®. Se consideró que existía asociación entre las variables y la presencia de la enfermedad cuando el valor de p era menor a 0.05 en la prueba de Chi Cuadrado. El mismo valor se empleó para considerar una variable como factor de riesgo en el análisis de Regresión Logística.

\section{Resultados}

Se trabajó con 5773 animales en los 17 caseríos. De estos, la tercera parte (1927/ 5773) era mayor de 7 meses de edad. El 97\% (1872/1927) de las muestras se analizó con la prueba de EITB, resultando 823 positivas, lo que representa una seroprevalencia de $44 \%(823 / 1872)$ con intervalos de confianza de $2.3 \%$. Sin embargo, la seroprevalencia encontrada fue corregida por la sensibilidad y especificidad de la prueba de EITB, lo que da un resultado de $45 \pm 2.3 \%$. Además, se

Cuadro 2. Distribución de la seroprevalencia de cisticercosis porcina en animales mayores de siete meses de edad en la provincia de Tumbes, Perú, según combinaciones de bandas reactivas a la prueba de EITB (2006)

\begin{tabular}{|c|c|c|c|}
\hline $\mathrm{N}^{\mathrm{o}}$ de bandas & Combinaciones de bandas & \multicolumn{2}{|c|}{$\begin{array}{l}\text { Total por banda } \\
\quad(\mathrm{n}=823)\end{array}$} \\
\hline 1 & $\begin{array}{l}50 \\
42-39\end{array}$ & $\begin{array}{c}233 \\
1\end{array}$ & 234 \\
\hline 2 & $\begin{array}{l}50,42-39 \\
42-39,24 \\
50,24 \\
50,13\end{array}$ & $\begin{array}{l}74 \\
47 \\
15 \\
22\end{array}$ & 158 \\
\hline 3 & $\begin{array}{l}50,42-39,24 \\
50,42-39,13 \\
42-39,24,13\end{array}$ & $\begin{array}{c}374 \\
5 \\
1\end{array}$ & 380 \\
\hline 4 & $\begin{array}{l}50,42-39,24,13 \\
50,42-39,24,21\end{array}$ & $\begin{array}{r}27 \\
1\end{array}$ & 28 \\
\hline 5 & $50,42-39,24,21,18$ & 2 & 2 \\
\hline 6 & $\begin{array}{l}50,42-39,24,21,18,13 \\
50,42-39,24,21,18,14\end{array}$ & $\begin{array}{l}5 \\
1\end{array}$ & 6 \\
\hline 7 & $50,42-39,24,21,18,14,13$ & 15 & 15 \\
\hline
\end{tabular}


Cuadro 3. Resultados de la Regresión Logística y Seroprevalencia de cisticercosis porcina en la provincia de Tumbes, Perú, según caserío de procedencia (2006)

\begin{tabular}{lcccccc}
\hline Caseríos & $\begin{array}{c}\text { Animales } \\
\text { muestreados }\end{array}$ & $\begin{array}{c}\text { Prevalencia } \\
(\%)\end{array}$ & $\begin{array}{c}\text { Odds } \\
\text { ratio } \\
(\mathrm{OR})\end{array}$ & $\mathrm{p}$ & $\begin{array}{c}\text { Intervalo de confianza } \\
\text { del OR (95\%) }\end{array}$ \\
\hline La Capitana & 132 & 70 & 12.04 & 0.00 & 5.55 & 26.09 \\
Carretas & 55 & 69 & 11.17 & 0.00 & 4.61 & 27.03 \\
Rica Playa & 192 & 64 & 9.01 & 0.00 & 4.30 & 18.89 \\
El Oidor & 94 & 64 & 8.52 & 0.00 & 3.83 & 18.93 \\
C. Blanqueada & 137 & 62 & 7.87 & 0.00 & 3.68 & 16.84 \\
Francos & 43 & 62 & 7.01 & 0.00 & 2.81 & 17.53 \\
Higuerón & 90 & 50 & 4.95 & 0.00 & 2.24 & 10.98 \\
Vaquería & 134 & 48 & 4.60 & 0.00 & 2.15 & 9.83 \\
Cristales & 46 & 38 & 3.00 & 0.02 & 1.21 & 7.43 \\
Malval & 113 & 36 & 2.88 & 0.01 & 1.32 & 6.30 \\
San Francisco & 49 & 35 & 2.64 & 0.04 & 1.08 & 6.50 \\
La Peña & 136 & 35 & 2.51 & 0.02 & 1.17 & 5.41 \\
Santa Rosa & 71 & 33 & 2.50 & 0.03 & 1.09 & 5.71 \\
El Rodeo & 80 & 33 & 2.45 & 0.04 & 1.06 & 5.70 \\
San Jacinto & 314 & 29 & 2.08 & 0.05 & 1.01 & 4.27 \\
Pechichal & 124 & 26 & 1.79 & 0.15 & 0.81 & 3.95 \\
Plateros & 62 & 17 & & & & \\
\hline Total & 1872 & 45 & & & & \\
\hline
\end{tabular}

observó un alto número de animales que reaccionaron a 3 bandas, a diferencia de animales que reaccionaron a 4 o más bandas (Cuadro 1).

En el Cuadro 2 se observa el número de animales positivos a la prueba de EITB que reaccionaron a una o más bandas, y las diversas combinaciones de bandas.

Se encontró asociación entre la variable caserío y la presentación de la enfermedad $(\mathrm{p}<0.05)$ con la prueba de Chi Cuadrado. Por esta razón, se realizó el análisis de Regresión Logística, ajustado por la edad y el sexo, para determinar el riesgo de cada caserío para la presentación de la enfermedad, tomándose al caserío de Plateros como el basal, por presentar el menor porcentaje de infección en comparación al resto de caseríos (Cuadro 3). Se observó que fuera de los caseríos de Pechichal y San Jacinto, los demás presentaron una mayor probabilidad de encontrar animales con cisticercosis porcina $(\mathrm{p}<0.05)$.

La seroprevalencia de animales mayores de 12 meses ( $49 \pm 3.1 \%$; 489/1026) fue mayor que aquella para animales de 8 a 12 meses de edad $(40 \pm 3.3 \% ; 334 / 846)(\mathrm{p}<0.05)$. El Odds Ratio de 1.52 para esta variable fue estadísticamente significativo $(\mathrm{p}<0.05)$. En 
relación al sexo, se encontró una seroprevalencia de $45 \pm 2.6 \%$ en hembras $(623 / 1413)$ y $45 \pm 4.5 \%$ en machos (200/459), indicativo de que no existe asociación alguna entre la variable sexo y la presentación de la enfermedad.

\section{Discusión}

El complejo teniasis/cisticercosis por Taenia solium está ampliamente distribuido en el Perú. La variabilidad en los niveles de infección es dependiente de muchos factores intrínsecos y extrínsecos al parásito, entre los que se encuentran la crianza libre de cerdos, la falta de uso de letrinas o su mal uso por parte del hombre, y el fácil acceso de los cerdos a las excretas humanas (González et al., 1999; Flisser, 2006).

La seroprevalencia corregida de $45 \%$ encontrada en el presente estudio fue similar al valor de $43 \%$ encontrado en Canchayllo, Junín (Morales, 1996) y en Maceda, San Martín (Castro, 1991), y superior al 30.8\% en Zarumilla, Tumbes (Mena et al., 2004), $35 \%$ en Cusco (Ramos, 1994) y $28 \%$ en Omnia, Amazonas (Carhuallanqui, 2007). La distribución de animales positivos según el número de bandas reactivas fue similar al estudio local de Quesquén (1999), quien, además, reportó que los animales que reaccionaron a 4 o más bandas fueron la minoría. El nivel de infección encontrado comprueba que la enfermedad persiste en un estado endémico en la zona de estudio, constituyendo un riesgo permanente para la salud pública, además de ocasionar pérdidas en el precio de la carcasa, afectando negativamente los ingresos económicos de los pobladores de estos caseríos.

Las muestras fueron analizadas mediante la prueba de EITB, la cual detecta anticuerpos de animales que presentan quistes viables en su musculatura, así como de animales que alguna vez presentaron la infección, pero que ya no la padecen, y de animales que presentan anticuerpos maternales
(González et al., 1999). Por esta razón la prueba de EITB es, en la actualidad, el mejor indicador para determinar exposición a huevos de Taenia solium (Tsang y García, 1999).

Los niveles de infección de la cisticercosis porcina en los caseríos endémicos en el país están entreel 20 al 42\% (Tbico et al., 2003). Los caseríos de La Capitana, Carretas y Francos pueden ser clasificados como hiperendémicos, toda vez que sus niveles de infección sobrepasan el $72 \%$, que es el nivel más alto reportado hasta el momento en el país por Bernal (1996) en Quilcas, Junín. Por otro lado, los caseríos restantes se encontrarían en estado de endemicidad, al tener niveles de infección mayores al 20\% (Taico et al., 2003). Se tiene un reporte en una zona cercana, Monte Redondo, Piura, con una baja prevalencia de 5.2\% (Gavidia, 1993).

Los caseríos que presentaron las seroprevalencias más altas como La Capitana, Carretas, Rica Playa, El Oidor, Casa Blanqueada, Francos, Higuerón y Vaquería, construyen letrinas elevadas del suelo (aprox. 30 $\mathrm{cm})$ con una entrada posterior para los cerdos, con la finalidad que consuman la materia fecal humana y de esta forma suplir la falta de alimento de los cerdos. Similares resultados han sido reportados por Bernal (1996) y Ayvar (2002), donde la presencia de la enfermedad es mayor en lugares donde se practica la crianza libre de cerdos y el fácil acceso de los cerdos a las heces humanas.

Existe evidencia que el riego de contraer la enfermedad aumenta con la edad (Taico et al., 2003; Mena et al., 2004). Esto fue comprobado en el presente estudio donde el análisis de Regresión Logística mostró una mayor probabilidad de encontrar casos positivos en animales mayores de 12 meses que entre aquellos en el rango de 8 a 12 meses de edad $(\mathrm{p}<0.05)$. Además, se ha reportado que la tasa de infección se encuentra homogénea hasta los 14 a 17 meses y empieza a aumentar a partir de los 22 meses (Bernal, 1996). No se tomó en cuenta animales menores de 8 meses en el presente 
estudio por la interferencia de anticuerpos maternales (Ccama et al., 2003).

La falta de asociación entre el sexo y la presentación de la enfermedad también ha sido observada en otros estudios (Ayvar, 2002; Carhuallanqui, 2007), aunque Mena et al. (2004) reportaron que los machos presentaron una mayor probabilidad de infectarse. Sin embargo, la literatura científica es concluyente en indicar que la presentación de la enfermedad no es afectada por el sexo.

El complejo teniasis/cisticercosis por Taenia solium puede ser monitoreado en la población humana o porcina. Sin embargo, es más factible realizarlo en la población porcina, debido a que los cerdos están constantemente muestreando el ambiente, donde pueden infectarse con huevos de $T$. solium (González et al., 1994).

\section{Conclusiones}

? La seroprevalencia de la cisticercosis porcina fue de $45 \%$ en los caseríos de Tumbes.

? Los caseríos que presentaron las seroprevalencias más altas fueron $\mathrm{La}$ Capitana, Carretas y Rica Playa con seroprevalencias de $70 \pm 7.8,69 \pm 12.3$ y $64 \pm 6.8 \%$, respectivamente.

? Se encontró que las variables zona de muestreo y edad representan factores de riesgo respecto a la presentación de la enfermedad.

\section{Limeratura Citada}

1. Acha P, Szyfres B. 2003. Zoonosis y enfermedades transmisibles, comunes al hombre y a los animales. Vol III. $3^{\mathrm{a}}$ ed. Publicación Técnica y Científica № 580. Washington DC: Organización Panamericana de la Salud. p 171-173.

2. Ahlbom A, Norell S. 1990. Introduction to modern epidemiology. $2^{\mathrm{d}}$ ed. USA: Resources Inc. 102 p.
3. Armitage P, Berry G. 1987. Statistical methods in medical research. $2^{\text {nd }}$ ed. Great Britain: Blackwell Scientific. 504 p.

4. Ayvar V. 2002. Seroprevalencia de la cisticercosis porcina en las villas de Nueva Esperanza, Matapuquio y Turpo en la provincia de Andahuaylas, departamento de Apurímac. Tesis de Médico Veterinario. Lima: Facultad de Medicina Veterinaria, Universidad Nacional Mayor de San Marcos. 48 p.

5. Bernal T. 1996. Evaluación de la cisticercosis porcina en el distrito de Quilcas - Huancayo. Tesis de Médico Veterinario. Lima: Facultad de Medicina Veterinaria, Universidad Nacional Mayor de San Marcos. 45 p.

6. Carhuallanqui M. 2007. Seroprevalencia de cisticercosis porcina en cuatro caseríos del distrito de Omia, Amazonas. Tesis de Médico Veterinario. Lima: Facultad de Medicina Veterinaria, Universidad Nacional Mayor de San Marcos. $94 \mathrm{p}$.

7. Castro V. 1991. Prevalencia de cisticercosis porcina: comparación de examen de lengua y EITB en Maceda, Tarapoto, departamento de San Martín. Tesis de Médico Veterinario. Lima: Facultad de Medicina Veterinaria, Universidad Nacional Mayor de San Marcos. 32 p.

8. Ccama A, González A, Falcón N, Bernal T. 2003. Persistencia de anticuerpos maternales contra cisticercosis porcina y su efecto en la interpretación de resultados del EITB. Rev Inv Vet, Perú 14: 140-144.

9. Cordero Del Campillo M, Hidalgo Argüiello M. 1999. Cisticercosis. En: Cordero Del Campillo M, Rojo Vázquez F (eds). Parasitología veterinaria. España: McGraw-Hill-Interamericana. p 493495.

10. [CWGP] The Cysticercosis Working Group in Peru. 1993. The marketing of cysticercotic pigs in the sierra of Peru. Bull World Health Organization 21: 223-226.

11. Del Brutto O. 1999. Neurocysticercosis. Rev Neurológica 29(5): 456-466. 
12. Flisser A. 2006. Epidemiología. En: Larralde C, De Aluja A (eds). Cisticercosis; guía para profesionales de la salud. México: Fondo de Cultura Económica, Secretaría de Salud, Instituto Nacional de Salud Pública, Fundación Mexicana para la Salud. p 87-103.

13. García H, González A. 2000. Teniasis por Taenia solium. Diagnóstico 39: 176178.

14. Gavidia C. 1993. Prevalencia de cisticercosis porcina en un pueblo de la costa norte: Monte Redondo (Piura). Tesis de Médico Veterinario. Lima: Facultad de Medicina Veterinaria, Universidad Nacional Mayor de San Marcos. 38 p.

15. González A. 2002. Control of Taenia solium with porcine chemotherapy. En: Singh G, Prabhakar S (eds). Taenia solium cysticercosis: From basics to clinical science. India: CABI Publishing. p 431-435.

16. González A, Gavidia C, Falcón N, Evans C, Bernal T, López T, García H, Gilman R. 1999. Porcine cysticercosis: epidemiology, diagnosis and treatment. En: García H, Martínez S (eds). Taeniasis/ Cysticercosis by Taenia solium. $2^{\mathrm{a}}$ ed. Perú: Ed Universo. p 97-120.

17. González A, Gilman R, García H, Mac Donald J, Kacena K, Tsang V, Pilcher $J$, et al.1994. Use of sentinel pigs to monitor environmental Taenia solium contamination. Am J Trop Med Hyg 51: 847-850.

18. González A, Ccama V, Gilman R, Tsang V, Pilcher J, Chavera A, Castro $M$, et al. 1990. Prevalence and comparison of serologic assays, necropsy, and tongue examination for the diagnosis of porcine cysticercosis in Peru. Am J Trop Med Hyg 43(2): 194-199.

19. [INEI] Instituto Nacional de Estadística e Informática. 2007. Censo Nacional XI de población y VI de vivienda. Lima, Perú.

20. Mena C, González A, Falcón N, Bernal T, Ayvar V. 2004. Incidencia de cisticercosis porcina en el distrito de Matapalo, Tumbes. Rev Inv Vet, Perú 15(1): 63-69.
21. Morales L. 1996. Seroprevalencia de cisticercosis porcina en la sierra central Canchayllo - Junín. Tesis de Médico Veterinario. Lima: Facultad de Medicina Veterinaria, Universidad Nacional Mayor de San Marcos. 38 p.

22. Náquira C. 1999. Taenia solium: Biological cycle and characteristics. In: García H, Martínez S (eds). Taeniasis/ Cysticercosis by Taenia solium. $2^{\text {nd }}$ ed. Perú: Ed Universo. p 7-14.

23. [OPS] Organización Panamericana de la Salud. 1992. El control de las enfermedades transmisibles en el hombre. Washington DC. Informe Oficial de la Asociación Estadounidense de Salud Pública. $651 \mathrm{p}$.

24. Portal Municipal Tumbes. 2008. Municipalidad Provincial de Tumbes. [Internet], [30 octubre 2008]. Disponible en: http://www.munitumbes.gob.pe/

25. Quesquén C. 1999. Correlación entre la prueba de electroinmuno transferencia blot (EITB) y la carga parasitaria en cisticercosis porcina. Tesis de Médico Veterinario. Lima: Facultad de Medicina Veterinaria, Universidad Nacional Mayor de San Marcos. 42 p.

26. Ramos U. 1994. Estudio de la prevalencia de cisticercosis porcina en SayllaCuzco. Tesis de Médico Veterinario. Lima: Facultad de Medicina Veterinaria, Universidad Nacional Mayor de San Marcos. 36 p.

27. Sartí E. 1997. La teniosis y cisticercosis por Taenia solium. Salud Pública Méx 39: 225-231.

28. Sciutto E, Hernández M, García G, De Aluja A, Villalobos A, Rodarte L, Parkhouse M, Harrison L. 1998. Diagnosis of porcine cysticercosis: a comparative study of serological tests for detection of circulating antibody and viable parasites. Vet Parasitol 14: 185-194.

29. Taico F, López T, González A, García H, Gilman R. 2003. Epidemiología de la cisticercosis porcina en tres caseríos de la provincia de Zarumilla, Tumbes. Rev Inv Vet, Perú 14: 166-173. 
30. Tsang V, García H. 1999. Inmunoblot diagnostic test (EITB) for Taenia solium cysticercosis and its contribution to the definition of this under-recognized but serious public health problem. In: García H, Martínez S (eds). Taeniasis/ Cysticercosis by Taenia solium. Section
III. $2^{\text {nd }}$ ed. Perú: Ed Universo. p 245-254.

31. Tsang VC, Brand JA, Boyer AE. 1989.

An enzyme-linked immunoelectrotransfer blot assay and glycoprotein antigens for diagnosing human cysticercosis (Taenia solium). J Infect Dis 159: 50-59. 